\title{
Chloride Channels of Intracellular Membranes
}

\author{
John C. Edwards* and Christina R. Kahl \\ UNC Kidney Center and the Division of Nephrology and Hypertension, Department of Medicine, \\ University of North Carolina at Chapel Hill
}

\begin{abstract}
Proteins implicated as intracellular chloride channels include the intracellular $\mathrm{ClC}$ proteins, the bestrophins, the cystic fibrosis transmembrane conductance regulator, the CLICs, and the recently described Golgi $\mathrm{pH}$ regulator. This paper examines current hypotheses regarding roles of intracellular chloride channels and reviews the evidence supporting a role in intracellular chloride transport for each of these proteins.
\end{abstract}

\section{Keywords}

chloride channel; ClC; CLIC; bestrophin; GPHR

\begin{abstract}
The study of chloride channels of intracellular membranes has seen enormous advances over the past two decades and exciting recent developments have sparked renewed interest in this field. The discovery of important roles for intracellular chloride channels in human disease processes as diverse as retinal macular dystrophy, osteopetrosis, renal proximal tubule dysfunction, and angiogenesis have highlighted the importance of these molecules in critical cellular activities. Startling discoveries regarding the intracellular $\mathrm{ClC}$ family of proteins have forced a re-examination of some of the fundamental assumptions regarding acidification of intracellular organelles. Newly discovered channels have attracted intense interest and the importance of long-recognized proteins has been questioned.
\end{abstract}

Investigations of intracellular channels present unique technical challenges. Perhaps most importantly, channels expressed exclusively on intracellular membranes are largely inaccessible to the direct application of the powerful patch-clamp techniques that led to rapid characterization of plasma membrane ion channels. As an alternative, intracellular channels can be studied in isolated vesicle fractions, but membrane fractionation techniques are always imperfect, with unavoidable contamination of membranes prepared from one organelle with those from other compartments. While low level contamination may not be critical to typical biochemical studies, contamination can be a fatal confounder in single molecule assays such as single channel recordings. These and other technical obstacles have impeded progress. Nonetheless, anion conductances have been demonstrated in numerous intracellular compartments and a host of discreet chloride channel activities have been described [1-3].

\footnotetext{
(C) 2010 Federation of European Biochemical Societies. Published by Elsevier B.V. All rights reserved.

*Address correspondence to: Campus Box 7155, Burnette-Womack 5020 Department of Medicine University of North Carolina at Chapel Hill Chapel Hill, NC, 27599-7155 Phone: (919) 966-2561 Fax: (919) 966-4251 jedwards@med.unc.edu .

Publisher's Disclaimer: This is a PDF file of an unedited manuscript that has been accepted for publication. As a service to our customers we are providing this early version of the manuscript. The manuscript will undergo copyediting, typesetting, and review of the resulting proof before it is published in its final citable form. Please note that during the production process errors may be discovered which could affect the content, and all legal disclaimers that apply to the journal pertain.
} 
Perhaps counter-intuitively, the regulation of concentration or amount of chloride itself within compartments has not been seen as the major role of these intracellular chloride channels. Instead, the key role of chloride permeability has been thought to be to function as a shortcircuiting conductance to allow transport by electrogenic cation transport mechanisms. For example, acidification of intracellular organelles by the electrogenic proton ATPase is recognized as a process that requires a short-circuiting conductance to allow transmembrane cation transport [4,5]. Other processes which may require a chloride short-circuiting conductance across intracellular membranes include calcium transport across the sarcoplasmic reticulum [3,6] and potassium influx into secretory vesicles [7].

While earlier investigations established the presence and possible roles of chloride conductances in intracellular organelles, more recent studies have tended to focus on identification of the molecular components responsible for these activities. Several proteins have now independently been implicated in intracellular chloride conductances, including $\mathrm{ClC}$ family members, CFTR, bestrophins, the CLIC family, and a recently described protein GPHR. In this review, we will first briefly consider some physiological functions for chloride channels of intracellular organelles and then examine data supporting the roles of each of the protein families listed above. Intracellular roles for ClCs, CFTR, and the bestrophins have been the subjects of recent reviews and will be summarized only briefly. Evidence for a role of CLICs as intracellular chloride channels will be examined in more detail. This review will not address mitochondrial porins or VDAC.

\section{Roles for Intracellular Chloride Channels}

\section{Acidification of intracellular compartments}

Most intracellular compartments maintain a steady-state $\mathrm{pH}$ somewhat more acidic than the cytoplasm, varying from about 6.5 in early endosomes and the Golgi apparatus to as low as 4.5 in mature lysosomes [8]. This low $\mathrm{pH}$ is implicated in numerous intraluminal events including dissociation of ligand/receptor complexes along the endosomal/recycling pathway, activation of hydrolytic enzymes in lysosomes, appropriate post-translational modification of secreted proteins in the Golgi and trans-Golgi network, and loading of neurotransmitter vesicles $[5,9,10]$. In addition to these intraluminal actions, acidification also appears to be essential for membrane traffic itself. Blocking acidification results in cessation of membrane traffic, perhaps due to an essential role for low luminal $\mathrm{pH}$ in membrane fusion events, for recruiting components of the fusion apparatus to the vesicle, or in modulation of transmembrane components of the fusion apparatus $[10,11]$.

Acidification of intracellular compartments primarily occurs through actions of the vacuolar proton ATPase acting in parallel with a chloride conductance [12]. The pump is electrogenic, moving a hydrogen ion across the membrane using the free energy released by hydrolysis of ATP. It is clear from consideration of the free energy available to the pump and estimates of luminal buffering power and membrane capacitance of typical vesicles that actions of the pump alone in the absence of other leak mechanisms would lead to generation of an electrical potential but no significant proton gradient [13]. The chloride conductance short-circuits the electrical potential and allows the pump to generate a $\mathrm{pH}$ gradient. Since the chloride conductance is essential to allow acid transport, several authors have suggested that regulation of the chloride conductance could be one means of regulating compartmental $\mathrm{pH}$ [14-16], but whether the chloride conductance contributes to active regulation of $\mathrm{pH}$ of intracellular compartments remains uncertain. Several independent studies have concluded that at least for phagosomes, lysosomes, and the Golgi, anion conductance is not limiting for acidification and that steady state $\mathrm{pH}$ is primarily regulated by proton pump and leak rates [17-21]. Although the role of the proton ATPase in vesicular acidification is firmly established, recent evidence that ClC-5 functions as a chloride-proton exchanger rather than a channel suggests that non-H-ATPase 
dependent acidification mechanisms also may contribute under certain circumstances [22, 23].

\section{Release of Ca from endoplasmic and sarcoplasmic reticuli}

Cycles of regulated release of calcium from sarcoplasmic reticulum (SR) via calcium-release channels followed by reuptake of calcium by a calcium ATPase are key steps in muscle contraction [6]. Similar processes occur across endoplasmic reticulum (ER) membranes in nonmuscle cells. Both calcium release and reuptake are electrogenic processes that require counterion movement to allow mass transfer of calcium. Chloride channels are present in the SR but their precise role is uncertain $[3,6,24]$. The major calcium release channel of SR is the ryanodine receptor. Calcium release via ryanodine receptor in various experimental models does not require the presence of chloride or other permeable anions [24]. Hence the counterion movement is thought to be carried by cations, primarily potassium, although a contribution by chloride in vivo has not been conclusively disproven. A recent theoretical analysis suggests that the cation counterion current may be carried by the ryanodine receptor itself by virtue of its low ion selectivity [24]. Active calcium uptake into the ER/SR by the calcium ATPase is also an electrogenic process that requires counterion movement that may be supplied by chloride channels [25].

\section{Exocytosis of secretory vesicles}

The roles of ion channels in exoctyosis have been reviewed in detail [7]. The mechanistic diversity of exocytosis among various models systems of interest defy easy generalization. In brief, chloride channels are known to be present in essentially all secretory vesicles that have been studied. Many of these vesicles acidify at least transiently along the exocytic pathway and one role of chloride conductances in these vesicles is to support acidification. This acidification may have particular exocytic-specific roles in individual cell types. For instance, acidification is important for loading of certain neurotransmitter vesicles [9] and for processing of insulin in secretory vesicles of pancreatic $\beta$ cells [26]. Furthermore, acidification supported by a chloride conductance appears to play a role in exoctyosis itself in pancreatic $\beta$ cells [7]. In contrast, mature zymogen granules of the exocrine pancreas are not acidic and exocytosis does not require acidification [27]. Zymogen granule membranes contain potassium and chloride conductances which are activated during exocytosis and inhibitors of these channels inhibit exocytosis. Exactly how these channels support exocytosis remains uncertain [7].

Chloride channels of mitochondria-Mitochondria contain anion channel activities in both the inner and outer membranes [28]. The primary conductance of the outer membrane, the Voltage Dependent Anion Channel (VDAC), is more properly thought of as a porin rather than a typical ion-selective channel and is responsible for movement of anions, cations, and non-electrolyte metabolites across the outer membrane [29]. Ion permeability of the inner membrane is tightly regulated and needs to be very low under ATP-synthesizing conditions to allow the $\mathrm{pH}$ and electrical gradient generated by electron transfer chain to drive ATP synthesis by the mitochondrial ATPase. None-the-less, the inner membrane does contain a chloride channel activity known as the inner membrane anion channel (IMAC) and single channel studies have identified discreet channel activities which may account for this conductance $[30,31]$ although the proteins responsible are unknown. The IMAC activity is thought to contribute, in parallel with a potassium conductance, to mitochondrial volume regulation [28] and to oxidative stress-related inner membrane depolarization [32]. A second anion conductance of the inner membrane is associated with the uncoupling protein UCP. UCP functions as a proton and chloride leak mechanism that uncouples the electron transport chain from ATP synthesis and leads to heat generation from mitochondria in brown fat [28]. Most recently, an inner membrane high conductance anion selective channel has been reported which 
is postulated to be related to the Permeability Transition Pore [33] although its role in development of this non-selective permeabilization is uncertain.

Other proposed functions-Other possible roles for intracellular chloride transporters have been proposed but in many cases the evidence supporting these roles are less well developed than those discussed above. For example, anion conductances have been proposed to support exit of superoxide from the lumen of endosomes [34] and mitochondria [35], to contribute to loading of nitrate into vacuoles in plants [36], and to allow ATP entry into Golgi membranes [37]. Novel, currently unrecognized roles of these channels are likely to be discovered as our understanding of intracellular metabolism becomes more and more sophisticated.

\section{Specific Chloride Channel Proteins in Intracellular Membranes}

\section{A brief note on evidence}

In each of the sections below, experiments will be discussed which attempt to identify individual chloride transporters as the protein responsible for counterion movement supporting electrogenic cation transport in specific intracellular compartments. Several types of evidence can be used to support these hypotheses. First, of course, it is important to know that the protein can function as an ion channel (or support electrogenic ion movement). Second, the protein should be present in the membrane fraction in which the transport is taking place. Third, chloride permeability of the membrane fraction in question should be reduced in the absence of the transporter. Fourth, the coupled cation transport (i.e., $\mathrm{H}^{+}$or $\mathrm{Ca}^{2+}$ flux) should be reduced in the absence or inhibition of the anion transporter. Fifth, cation transport in the absence of the chloride transporter should be rescued by provision of an alternative counterion transport pathway (e.g., the potassium ionophore valinomycin in the presence of potassium). The last point is quite important: if cation transport is not rescued by an alternative short-circuiting mechanism, then the inhibition of transport is not simply due to electrical potential limiting the cation transport mechanism; the chloride transporter must be doing something other than simply short circuiting the membrane. In fact, for none of the proteins discussed below does the current data fully satisfy all five criteria, leaving real uncertainty about the true role of the chloride transporter in each case.

\section{CIC Family Proteins}

The strongest and most consistent evidence supporting particular proteins as intracellular chloride transporters apply to the $\mathrm{ClC}$ family of proteins. Since these proteins are the subject of several recent extensive reviews [37-40], these data will only be briefly summarized here. The $\mathrm{ClC}$ family consists of 9 separate genes that can be grouped in three clusters based on homology and function. $\mathrm{ClC}-1, \mathrm{ClC}-2, \mathrm{ClC}-\mathrm{Ka}$, and $\mathrm{ClC}-\mathrm{Kb}$ are expressed on the plasma membrane where they function as chloride channels. Extensive physiologic, biochemical and genetic studies have firmly established that these proteins are responsible for plasma membrane chloride channel activities. Two other clusters, consisting of ClCs 3 through 5, and ClCs 6 and 7 , are expressed primarily in intracellular membranes. Intracellular ClCs show compartmentspecific expression patterns with $\mathrm{ClC}-5$ predominant in early and recycling endosomes, $\mathrm{ClC}-3$ and $\mathrm{ClC}-4$ in later and sorting endosomes, and $\mathrm{ClC}-6$ and 7 in late endosomes, lysosomes, and the osteoclast ruffled membrane.

\section{Functional Roles for Intracellular CICs}

Both engineered $\mathrm{ClC}$ mutations in mice and naturally occurring mutations in humans strongly support a role for intracellular $\mathrm{ClCs}$ in various intracellular membrane functions. Mutations in ClC-5 are the basis for Dent's disease, a renal defect associated with low molecular weight 
proteinuria, hyperphosphaturia, hypercalicuria, and aminoaciduria [40]. Much of this phenotype is reproduced in ClC-5 knockout mice [41,42]. ClC-5 is expressed primarily in the renal proximal tubule where it colocalizes with markers of early apical endosomes [43]. Proximal tubule cells from ClC-5 knock-out mice show impaired apical membrane traffic [44] and decreased endosomal acidification [45], but whether the defect in acidification is due to limited counterion movement has not been resolved. The entire phenotype of Dent's disease can be explained by consequences of failure of apical early endosomes to acidify, resulting in reduced proximal tubule endocytosis and membrane traffic, which in turn disrupts clearance from the tubular lumen of low molecular weight proteins including PTH and the vitamin D/ vitamin $\mathrm{D}$ binding protein complex [40].

$\mathrm{ClC}-3$ is expressed in endosomes in many cell types and in synaptic vesicles. Endosomes or synaptic vesicles lacking $\mathrm{ClC}-3$ have decreased acidification and decreased chloride permeability [46]. Mutations in ClC-3 in mice result in neurodegeneration [38]. ClC-4 is also expressed along the endosomal pathway. Cells from ClC-4 knockout mice have been shown to have reduced endosomal acidification and consequent defects in recycling of the transferrin receptor [47]. As with ClC-5, the defective acidification seen in the absence of either ClC-3 or $\mathrm{ClC}-4$ has not been shown to be due to absence of counterion permeability. ClC-6 is expressed in late endosomes in the nervous system and disruption of ClC-6 results in neurodegeneration with pathological features of lysosomal storage disease [38].

ClC-7 is expressed in late endosomes and lysosomes of many cell types and in the osteoclast ruffled membrane. Disruption of ClC-7 causes a complex phenotype including neurodegeneration and renal dysfunction with pathological findings of a lysosomal storage disease consistent with a disruption of lysosomal function [48]. Supporting a role in lysosomes, a recent rigorous study demonstrated that $\mathrm{ClC}-7$ accounts for the major chloride permeability of lysosomes [49]. More prominently, ClC-7 knock-out mice also show severe osteopetrosis [50]. Osteoclasts are present but have poorly developed ruffled membrane and fail to generate an acidic bone resorption compartment or resorb bone. These observations lead to the hypothesis that $\mathrm{ClC}-7$ is responsible for counterion current that allows acid transport across the osteoclast ruffled border, but absence of counterion movement as the cause of the acidification defect has not been demonstrated.

Some forms of inherited human osteopetrosis carry mutations in ClC-7 [51] and osteoclasts derived from such patients show defective bone resorption [52,53]. A recent study directly assessed acid transport by vesicles derived from dominant-negative ClC-7 mutant and control human osteoclasts [53]. Peripheral blood cells were purified and induced to differentiate into osteoclasts in culture. Membranes were prepared from these cells and assayed for acidification using an acridine orange quenching assay carried out in the presence of $\mathrm{K}^{+}$and valinomycin which would provide robust counterion movement independent of any chloride transport mechanism. As expected, membranes from control cells acidified and the acidification was inhibited by an inhibitor of the proton pump, but surprisingly, acidification was also inhibited by an inhibitor of ClC-7. Vesicles prepared from mutant cells had equivalent levels of proton pump and ClC-7 protein, but showed significantly decreased acidification rate. Unfortunately, the rates of acidification in the absence of valinomycin were not reported. The interpretation of these data is complex. The inhibition of acid transport in the control cells by a $\mathrm{ClC}$ inhibitor and the decreased acid transport by the mutant cells, both in the presence of $\mathrm{K}^{+} / \mathrm{valinomycin}$ indicate that $\mathrm{ClC}-7$ is important in osteoclast acid transport, but also indicate that inhibition of transport is not due to lack of counterion movement. Although the authors of the paper came to a different conclusion, a valid interpretation of this data is that these results challenge the hypothesis that $\mathrm{ClC}-7$ provides the counterion anion conductance to allow acidification of the bone resorption compartment. 


\section{Intracellular $\mathrm{CICs}$ are Proton-Chloride Exchangers Rather than Chloride Channels}

Much of the early work with intracellular $\mathrm{ClCs}$ was based on the assumption that these proteins produce chloride conductive pores similar to the cell surface $\mathrm{ClC}$ family members. Consequently the discovery that $\mathrm{ClCs} 4$ and 5 function not as channels but as electrogenic proton-chloride exchangers was a surprising and unsettling event [22,23]. Based on identity at a critical glutamate residue in the sequence, it seems very likely that all that intracellular $\mathrm{ClCs}$ 3 through 7 are indeed exchangers rather than channels $[38,40]$. The stoichiometry of ion exchange is thought to be approximately $2 \mathrm{Cl}^{-}$for $1 \mathrm{H}^{+}$for $\mathrm{ClCs} 4,5$, and 7 [22,23,49].

Could a proton-chloride antiporter account for the counterion movement necessary for the acidification of intracellular compartments by the proton ATPase? As long as the net negative charge movement into the vesicle is greater than the proton efflux with each cycle of the exchanger, such activity could provide the necessary counterion movement and allow acidification. Indeed, since most transport experiments demonstrating apparent chloride channel activity supporting acidification were performed in buffered solutions, a protonchloride exchange nature of the counterion current may well have gone unnoticed in the older studies. However, several considerations must give one pause about this mechanism. First, such a mechanism would be wasteful of metabolic energy since one third of the protons pumped into the vesicle by the ATPase would need to come back out in exchange for chloride (assuming a stoichiometry of $2 \mathrm{Cl}^{-}$for $1 \mathrm{H}^{+}$). While this does not rule $\mathrm{Cl}^{-} / \mathrm{H}^{+}$exchange as the counterion mechanism, it suggests some other unrecognized secondary gain exists to justify the apparent waste. A plausible explanation for why the cell would employ this apparently wasteful strategy would increase confidence that this hypothesis is correct. Second, the strong outward rectification of $\mathrm{Cl}^{-} / \mathrm{H}^{+}$exchange $[22,23]$ indicates that the charge-compensating transport would be occurring under conditions where the transporter activity is low. Again this does not rule out the mechanism, but it suggests that $\mathrm{Cl}^{-} / \mathrm{H}^{+}$exchange would be most active under conditions and in a polarity where it is not needed. Third, although defective acidification of various intracellular compartments occurs with defects in individual $\mathrm{ClCs}$, there has been no direct demonstration that the acidification defect is due to a loss of counterion movement. Finally, despite the apparent lysosomal storage disease phenotype in ClC-6 or ClC-7 knockout mice, the steady state lysosomal $\mathrm{pH}$ in these mice has been reported to be normal [48] (although a second group using semi-quantitative methods reported less lysosomal acidification following RNA-i induced knock-down of ClC-7 in cultured cells [49]). The failure of loss of a single protein to prevent acidification is not too surprising due to the possibility of functional redundancy among closely related proteins. For example, since their subcellular distribution overlaps, ClC-6 could conceivably compensate for the absence of $\mathrm{ClC}-7$ and vice versa. However in the absence of an acidification defect, the lysosomal storage phenotype must be due to something other than failure to maintain the low steady state $\mathrm{pH}$ of lysosomes and hence, perhaps the primary role of $\mathrm{ClC}-7$ is something other than support of lysosomal acidification.

Alternative functions for $\mathrm{ClCs}$ have been hypothesized. First, $\mathrm{ClCs}$ in early endosomes could provide a proton-pump-independent acidification mechanism [22,23]. Immediately after formation of the endosome, the intravesicular $\left[\mathrm{Cl}^{-}\right]$(about $110 \mathrm{mM}$ ) would be much higher than the cytoplasmic $\left[\mathrm{Cl}^{-}\right]$(about 10-40 mM). This gradient could drive chloride-proton exchange in the opposite polarity as that hypothesized to occur above, leading directly to vesicular acidification and lowering intravesicular $\left[\mathrm{Cl}^{-}\right]$. Although an attractive hypothesis, no evidence for such a mechanism contributing to endosomal acidification has been reported. Second, ClCs could be functioning as the proton leak mechanism which contributes to determination of steady state $\mathrm{pH}$ of intracellular compartments [22]. However, such a role would be difficult to reconcile with the observed alterations of vesicular $\mathrm{pH}$ in cells carrying mutations of $\mathrm{ClCs} 3-5$. If these proteins provided the proton leak mechanism, inactivation of 
this pathway should result in lower compartment $\mathrm{pH}$, not higher as observed. Third, ClCmediated $\mathrm{Cl}^{-} / \mathrm{H}^{+}$exchange could play an important role in maintaining elevated intravesicular chloride concentration and that alterations in intra-lysosomal $\left[\mathrm{Cl}^{-}\right]$rather than $\mathrm{pH}$ may be responsible for the lysosomal functional defects in ClC-6 and -7 mutants [38,40,48]. Finally, multiple other possible roles for ClC-5 in renal proximal tubule cells have been postulated [54] including the assembly of the macromolecular endocytic comples on the plasma membrane, trafficking of the proton ATPase, and regulation of megalin/cubulin expression. Whether any of these are direct functions of intracellular $\mathrm{ClCs}$ remain uncertain.

In summary, a robust body of evidence supports a role for the intracellular $\mathrm{ClCs}$ in supporting acidification of intracellular compartments. Whether their primary function is to provide the counterion movement allowing electrogenic acidification by the proton pump remains unproven. Integrating the ion exchange activities of these proteins into a comprehensive picture of intracellular ion movement consistent with the large body of experimental data continues to be a work in progress.

\section{Bestrophins}

The bestrophins are a group of 4 proteins (recently reviewed in $[55,56]$ ) which have been shown to support chloride channel activity when over-expressed in cell expression systems. Further evidence that bestrophins themselves function as channels stems from mutagenesis studies in which mutation of the bestrophin causes discrete changes in the associated channel activity. Bestrophin 1, the first member of the family to be discovered, was identified as the site of mutation in Best's disease, a form of early onset macular degeneration $[55,56]$. The function of bestrophin 1 relevant to the disease is to support a $\mathrm{Ca}^{2+}$-activated chloride current in the basal membrane of the retinal pigment epithelium but whether bestrophin 1 itself functions as this channel has been challenged [57]. Recently, an exciting alternative mechanism has been proposed by Kunzelmann and colleagues based on studies of bestrophin in airway epithelial cells [58]. These authors confirmed that endogenous bestrophin 1 primarily resides in the endoplasmic reticulum, not the plasma membrane. Furthermore they demonstrated that bestrophin 1 modulates both $\mathrm{Ca}^{2+}$ release and uptake from intracellular stores, presumably ER. Thus, bestrophin can modulate plasma membrane $\mathrm{Ca}^{2+}$-activated channels by its effects on ER $\mathrm{Ca}^{2+}$ release and recovery. These observations make bestrophin a strong candidate for an ER chloride channel that could provide counterion movement during cycles of $\mathrm{Ca}^{2+}$ release and reuptake [56]. However, some reservations exist regarding this conclusion. First, humans suffering from Best's disease who carry the inactivating bestrophin 1 mutation have little phenotype other than macular degeneration. Similarly, bestrophin 1 knock-out mice have little phenotype [59]. Thus, if bestrophin 1 serves as an ER counterion channel, it seems unlikely to be the only one; there must be significant functional redundancy with other counterion channels to support ER calcium transport in the absence of bestrophin. Second, bestrophin 1 appears to be primarily expressed in epithelial cells and expressed only in low levels in heart or skeletal muscle [55]. This expression pattern plus the absence of muscle phenotype in human or mouse mutants suggest that a different protein must play this role in muscle cells. Whether this function in muscle could be supported by a different bestrophin family member remains is unknown. Finally, the evidence so far that bestrophin is functioning as a channel in the ER is indirect [58]; the published evidence only demonstrates that it facilitates $\mathrm{Ca}^{2+}$ movement. The data is also consistent with a model in which bestrophin regulates ER calcium transport via mechanisms other than counterion movement. Direct demonstration of changes in ER anion conductivity following manipulation of bestrophin expression is an important missing piece of evidence. 


\section{CFTR}

Mutations in the cystic fibrosis transmembrane conductance regulator (CFTR) are the cause of the disease cystic fibrosis. CFTR functions as a cAMP activated plasma membrane chloride channel and also as a regulator of other channels and transporters. CFTR has also been hypothesized to function as a chloride channel in intracellular organelles, where it could contribute to support of acidification along the exocytic and endocytic pathways [14]. This hypothesis is particularly attractive as it could explain several peculiar features of the cystic fibrosis phenotype that at least on first blush do not appear to be related directly to defects in ion transport. A great deal of evidence supporting and contesting this hypothesis has been published over the years. Recently this hypothesis has been rigorously tested experimentally [17] and the published data has been extensively and critically reviewed [8], both approaches coming to similar conclusions. To summarize their findings, while CFTR may function as a chloride channel in some intracellular membranes, the bulk of the evidence indicates that organellar acidification is not dependent on CFTR and that organellar $\mathrm{pH}$ is not abnormal in cystic fibrosis. Recent evidence suggests a novel intracellular mechanism for CFTR in the regulation of endocytosis and apical membrane trafficking in renal proximal tubule cells [60]. What other roles CFTR may be playing in intracellular membranes and whether alterations in these functions contribute to the defects in cystic fibrosis remain uncertain.

\section{CLIC Family Protiens}

The CLICs are another group of proteins proposed to function as intracellular chloride channels. CLICs are a family of proteins encoded by 6 different genes in mammals, named CLICs 1 through $6[61,62]$. The proteins show high degree of similarity through the C-terminal approximately 220 amino acid CLIC homology domain which is structurally related to glutathione $\mathrm{S}$ transferases. The $\mathrm{N}$-termini of the proteins are divergent in both length and sequence.

The evidence that CLICs can function as channels is robust: the first CLIC to be described (p64, now known as CLIC5B) was identified based on purification of reconstitutable chloride channel activity from bovine kidney [63]. CLIC5B co-purified with channel activity and antibodies to the protein immunodepleted this activity. Expression of recombinant CLIC5B in cultured cells led to increased channel activity in whole cell membrane preparations and modification of the protein altered the activity [64,65]. Overexpression of CLICs 1, 3, and 4 were associated with chloride channel activity in a variety of expression systems at the times of their discovery [66-68]. More recently, several groups have reported ion channel activity from purified recombinant CLICs including CLICs 1, 2, 4, and 5 expressed in E. coli, although there is limited consensus on single channel conductance, ion selectivities, lipid requirements, and effects of oxidation among the published reports [69-76].

One of the problems confounding our understanding of CLIC function is their unusual biochemical properties. Unlike typical integral membrane proteins, CLICs exist both in a soluble and a membrane-inserted form. The majority of the CLIC in most resting cells is present as a soluble protein in the cytoplasm. However, some fraction of the CLIC is membraneassociated and shows the biochemical properties of truly membrane inserted protein. In vitro, purified recombinant soluble CLICs 1, 2, 4, and 5 [70-74,76] are capable of transition from the aqueous phase into a phospholipid membrane where they can function as ion channels. Control of the distribution of CLICs between soluble and membrane-inserted forms is clearly central to any understanding of how CLICs function, but very little is currently known about regulation of this critical process. 


\section{CLICs in Intracellular Membranes}

CLICs were among the first proteins proposed to function as intracellular chloride channels [77]. Indeed the name CLIC was derived from "Chloride Intracellular Channel," [78] but this is clearly a case where the name implies much more certainty than exists in reality. In most cases the evidence supporting the hypothesis that CLICs function as intracellular chloride channels has never moved much beyond the circumstantial. With the original report of CLIC5B sequence, it was noted that the protein is excluded from the plasma membrane when expressed in Xenopus oocytes [77]. As other CLICs were discovered, immunolocalization to a variety of intracellular compartments was reported. Thus, CLIC1 (also named NCC27) was reported to be in nuclear membrane and in an undefined cytoplasmic vesicular compartment [68,79]. CLIC4 (also called p64H1 and mitochondrial CLIC) was variously reported to be in endoplasmic reticulum, trans-Golgi network, caveolae, mitochondria, and dense-core secretory vesicles in the central nervous system $[65,66,80,81]$. CLIC5A was found to be in a sub-apical domain of placental trophoblast [82] and CLC5B was reported in osteoclast ruffled membrane $[83,84]$.

It is now clear that many of these immunolocalization reports were confounded by the abundant soluble cytoplasmic CLIC that obscures the true subcellular localization of the membraneinserted fraction of the protein. Ulmasov et al. used digitonin to extract selectively the soluble fraction of endogenous CLIC1 in Panc1 and T84 cells while leaving the membrane-inserted fraction in place [85]. Perhaps not surprisingly, the overall distribution of CLIC1 in Panc1 cells changed dramatically after removal of the soluble fraction which represents a majority of the CLIC1 in those cells. The punctuate pattern that had been interpreted as representing intracellular vesicles was eliminated, leaving the bulk of the residual CLIC1 in the plasma membrane. In contrast, extraction of the soluble CLIC1 from polarized T84 cells, where a larger fraction of the protein is membrane inserted, had minimal effect on the immunolocalization of the protein which was found in sub-apical membrane vesicles. Thus, the extent that soluble CLIC confounds the identification of membrane compartments in which the membrane inserted CLIC resides is cell-type dependent. Many of the earlier immunolocalization reports will need to be re-examined in light of this evidence.

In contrast to the abundant reports of morphologic data indicating that CLICs reside in intracellular membranes, the evidence for CLICs functioning as chloride channels in intracellular membranes is much more limited. In brief, there are three systems for which substantial evidence of CLIC chloride channel function in intracellular membranes have been reported: CLIC1 in nuclear membranes, CLIC5B in osteoclast ruffled membrane, and CLIC4 in vesicles along the intracellular tubulogenic pathway.

\section{CLIC1 in nuclear membrane}

The initial report of CLIC1 (then called NCC27) by Breit and colleagues reported nuclear localization in CHO-K1 cells and the appearance of increased chloride channel activity in both nuclear membranes and plasma membrane on expression of exogenous CLIC1 [68]. This activity showed similar single channel properties in both membrane fractions and was subsequently found to be similar to channel activity of purified recombinant CLIC1 in vitro [76]. Antibodies against a FLAG-tagged CLIC1 construct inhibited this activity in the plasma membrane. The plasma membrane activity associated with CLIC1 expression was strongly correlated with the cell cycle, being highest in G2/M phase [86]. Growth in the presence of the CLIC inhibitor, IAA94, arrested the cells in G2/M phase. Taken together, these data indicate CLIC1 supports chloride channel activity in both nuclear and plasma membranes and its activity is important to passage through the cell cycle. The inhibition of activity by antibodies against CLIC and the similarity between activity in cells and purified recombinant CLIC1 strongly support the contention that the activity is due to the CLIC protein itself and not to 
regulation of different channel protein. However, prominent nuclear localization of CLIC1 has only been reported in CHO cells, where the CLIC1 localization was more consistent with nucleoplasm rather than nuclear envelope localization. Examination of numerous other tissues and cell types have failed to find significant nuclear membrane CLIC1 [79,85]. In addition, a physiologic role for a chloride conductance in the nuclear membrane has never been clearly articulated, making it difficult to predict what the consequences of disrupting such a conductance would be. Thus, whether CLIC1 functions as a chloride channel in the nuclear membranes of most normal cells remains uncertain.

\section{CLIC5B in the osteoclast ruffled membrane}

A second role supported by substantial evidence for CLIC chloride channel activity in intracellular membranes is that of CLIC5B in the osteoclast ruffled membrane. The ruffled membrane is a specialized proton-transporting membrane that acidifies the bone resorption compartment beneath a bone-attached osteoclast. As with other intracellular membranes, the mechanism of acidification is the electrogenic proton ATPase coupled with a chloride conductance, together supporting transmembrane transport of $\mathrm{HCl}$ into the bone resorption compartment.

Several lines of evidence support a role for CLIC5B in bone resorption by osteoclasts [83, 84]. CLIC5B mRNA was found to be present in avian osteoclasts and the abundance of the message and protein are upregulated as mononuclear precoursor cells differentiate into boneresorbing osteclasts in culture. Suppression of expression of CLIC5B in differentiating osteoclasts using antisense oligonucleotides decreased bone resorption. Membrane vesicles from cells in which CLIC5B had been suppressed showed decreased acidification that was due to decreased counterion movement while total proton pump activity was unchanged. Thus, expression of CLIC5B during osteoclast differentiation supports bone resorption and is necessary for the presence of chloride counter-ion movement in proton-pump containing vesicles. Osteoclast activation is at least partially mediated through the c-src tyrosine kinase and absence of c-src suppresses bone resorption by osteoclasts. CLIC5B is phosphorylated by src-family tyrosine knases and and phosphorylation activates the channel activity in cultured cells overexpressing CLIC5B [64]. Suppression of c-src in differentiating osteoclasts led to loss of co-localization of CLIC with the proton ATPase concomitant with the loss of shortcircuiting chloride transport activity in proton-pump containing vesicles [83]. This set of experiments is unique in that knock-down of a chloride channel protein was not only shown to decrease acidification, but that the cause of decrease in acidification was clearly demonstrated to be the loss of counterion movement.

Taken together, these data indicate that CLIC5B is essential for the establishment of a chloride conductance in the proton pump-containing membranes of differentiating osteoclasts and appears to be a component of the signal transduction mechanisms that allow c-src to drive osteoclast differentiation and bone resorption. Perhaps the simplest interpretation of these data is that CLIC5B itself provides the ruffled membrane chloride conductance. An alternative hypothesis, equally consistent with the data, is that CLIC5B may be essential for assembly of the ruffled membrane rather than the function of the mature membrane. Other data implicate ClC-7 as providing the necessary counterion movement in the ruffled membrane as described earlier. Both CLIC5B and ClC-7 appear to play non-redundant roles in the assembly and/or function of the ruffled membrane. Further investigations will be necessary to elucidate the distinct roles of CLIC5B and ClC-7 in osteoclasts.

\section{CLIC4 in cell-hollowing tubulogenesis}

One mechanism by which cells form multicellular tubes is known as "cell-hollowing" in which an intracellular lumen forms within a single cell [87]. The lumen, which is like a large 
intracellular vacuole, forms from the fusion of numerous precursor intracellular vesicles. With ongoing vesicle fusion, the vacuole enlarges, eventually fusing with the plasma membrane, and in continuity with neighboring cells, forms an extracellular space that becomes the lumen of the cell-lined tubule. Thus a single cell forms a tubule by hollowing itself out. Cell-hollowing tubulogenesis contributes to tube formation in several structures including the C. elegans excretory system and mammalian capillary tubes.

The excretory system of $C$. elegans consists of a single excretory cell which has long extensions that reach to each end of the worm [88]. Within this cell, a single intracellular lumen called the excretory canal runs the length of the cell and connects to the exterior environment through duct and pore cells. The formation of this canal is one example of cell-hollowing tubulogenesis. Mutations in EXC4, one of the C. elegans CLIC genes, disrupts the structure of the excretory cell [88]. Worms with EXC4 disruption fail to generate the excretory canal, but instead accumulate a large number of unconnected dilated cysts. Using a temperature sensitive construct, Berry et al. [88] showed that introduction of functional CLIC protein after the cysts had formed results in resolution of the cysts into an intact canal. These studies indicate that the formation and persistence of the excretory canal requires the presence of the nematode CLIC homolog, apparently by permitting the fusion of intracellular precursor vesicles to form the intact canal. Thus, a CLIC molecule appears to be essential for cell hollowing tubulogenesis in this simple system, perhaps through facilitating fusion of intracellular membranes.

Formation of capillary tubes by endothelial cells is another example of cell-hollowing tubulogenesis. Using a proteomics approach, Bohman et al. identified CLIC4 as a protein which was strongly regulated during endothelial cell tube formation in culture [89]. Antisense knockdown experiments demonstrated a significant decrease in the ability of the cells to form tubule structures when expression of CLIC4 was suppressed. A second study confirmed a role for CLIC4 in endothelial tubulogenesis using CLIC4 overexpression and knockdown in cultured cells [90]. CLIC4 was found to be important for endothelial cell proliferation and tubulogenesis but not migration.

Recently my colleagues and I reported studies using a mouse in which the gene for CLIC4 had been disrupted [91]. The $\mathrm{Clic}^{-/-}$mice showed an increased rate of intra-uterine death but otherwise demonstrated minimal phenotype in unstressed laboratory conditions. When stimulated for maximal angiogenesis, the ability to form new blood vessels was markedly impaired. Reminiscent of the observations in C. elegans, accumulation of what appeared to be large unfused vesicles were noted in the tubulating endothelial cells in the absence of CLIC4. Primary cultures of endothelial cells were isolated from $\mathrm{Clic}^{-/-}$and wild-type littermate controls and induced to undergo tubulogenesis in fibrin gels. CLIC4 was immunolocalized to the limiting membranes of the vacuoles in WT cells. The $\mathrm{Clic}^{-/-}$cells showed impaired vacuolization with accumulation of unfused vacuoles. Vacuolization of WT cells was inhibited both by the proton pump inhibitor, Bafilomycin, and by the CLIC inhibitor, IAA94. Ratiometric fluorescence confocal microscopy was used to assess the $\mathrm{pH}$ of both the large vacuoles and the much smaller endosomal/lysosomal vesicles in tubulating cells. Vacuoles in WT cells showed modest acidification with average $\mathrm{pH}$ of 6.75 while the vacuoles of $\mathrm{Clic}^{-/-}$cells failed to acidify (average $\mathrm{pH} 7.05$ ). In contrast, the small vesicle fraction acidified well in both cell types with average $\mathrm{pH}$ of 5.42 and 5.43, respectively. Thus the absence of CLIC4 results in the selective failure to acidify vacuoles along the endothelial tubulogenic pathway. These data represent the first direct demonstration that the absence of a CLIC protein results in failure to acidify an intracellular compartment in vivo. These data support the hypothesis that CLIC4 itself could be functioning as the short-circuiting chloride conductance allowing acidification of this intracellular compartment. However, these experiments have neither showed that the chloride permeability of the vacuole membranes was altered or that the acidification failure was due to decreased counterion movment as would be expected if CLIC4 was acting as a 
chloride channel in these membranes. Hence, multiple other mechanisms by which CLIC4 could be supporting acidification are consistent with the data.

In summary, CLIC family members are implicated in intracellular chloride channel activity in numerous systems and cell types. However, definitive attribution of a specific channel activity of normal cells to a specific CLIC protein remains elusive. In the case of nuclear CLIC1, the evidence for CLIC1 channel activity in nuclear membranes when the recombinant protein is overexpressed in $\mathrm{CHO}$ cells is persuasive, but it is unclear whether these findings apply to more normal cells and conditions. In the osteoclast and endothelial cell models, CLIC5B and CLIC4 are clearly involved in establishment of acidification capacity, but whether they themselves function as the short-circuiting chloride conductance in these membranes, or support acidification by some other mechanism is unresolved.

\section{Non-channel roles of CLICs}

Evidence suggests that some CLIC family members, particularly CLIC4, possess important, non-channel roles in several different cell types. CLIC proteins are implicated in cell cycle regulation, cell differentiation, and apoptosis in various systems. They exist as both membraneinserted proteins and as soluble cytoplasmic proteins and their subcellular localization changes in response to extracellular stimuli in several cell types. While some studies have implicated CLIC4 and its regulated nuclear targeting in the apoptosis and the differentiation of fibroblasts into myofibroblasts, particularly as related to cancer [92-95], perhaps the most compelling data for a non-channel role of a CLIC applies to CLIC4 in TGF $\beta$ signaling during keratinocyte differentiation $[94,96]$. Shulka et al. reported TGF $\beta$ stimulation induces the expression and nuclear translocation of CLIC4, and that Schnurri, another protein involved in the TGF $\beta$ pathway, interacts with CLIC4 and promotes its nuclear translocation [94]. The transcription factors Smad2 and Smad3 are phosphorylated in response to TGF $\beta$ and translocate to the nucleus [97]. By immunoprecipitation, CLIC4 interacts with phosphorylated Smad2 and Smad3, and overexpression of a nuclear targeted CLIC4 protects Smad2 and Smad3 from dephosphorylation in keratinocytes [94]. Thus nuclear CLIC4, by protecting Smad2/3 from dephosphorylation, functions to potentiate TGF $\beta$-driven gene expression. These studies are the first to establish a non-channel mechanistic role for CLIC4 within the cell nucleus. It remains to be seen whether this pathway is specific to TGF $\beta$ signaling in keratinocytes or if CLIC4 plays a more global role in TGF $\beta$ signaling in other cell types. As CLIC4 is found in many subcellular locations, it will also be interesting to know if a specific pool of CLIC4 proteins, cytosolic or membrane associated, translocate to the nucleus following a specific stimulus.

Numerous binding partners have been identified for CLIC family members through different screening strategies but the functional significance for CLIC or its binding partners remains uncharacterized in many circumstances. The evidence that CLIC4 plays a role in the phosphorylation status of Smad 2 and Smad3 is intriguing since CLIC1 copurifies with protein phosphatase 1 (PP1 $\gamma 2$ ) in sperm extracts [98]. Fusion proteins of CLIC1, CLIC4, and CLIC5 all interact with PP1 $\gamma 2$ from sperm extracts in pull-down assays. In addition, studies demonstrate that CLIC3 can interact with ERK7, a mitogen-activated protein kinase, in mammalian cells [67]. CLIC proteins may regulate the phosphorylation status of substrates, the activity of the phosphatases or kinases, or the interaction may regulate CLIC function or localization through phosphorylation or dephosphorylation.

CLIC family members interact with multiple cytoskeletal elements but the importance of these interactions remains unclear. When recombinant CLIC5 and CLIC1 are inserted into lipid bilayers, their activity is inhibited by F-actin in the absence of other proteins [73]. In contrast, F-actin has no effect on CLIC4 activity. In a recent study, cytosolic CLIC4 was shown to translocate to the plasma membrane in response to $\mathrm{G}_{13}$-mediated RhoA activation and 
latrunculin inhibits this translocation, implying that this process requires F-actin [99]. Multiple CLIC proteins interact with AKAP350, which acts as a scaffolding protein for numerous other proteins including kinases and phosphatases [100,101]. Detailing all the binding partners of CLIC family members is beyond the scope of this review, but additional binding partners for CLICs include other cytoskeletal elements and cellular receptors. Taken together, these observations suggest CLICs are intimately involved in cytoskeletal and membrane trafficking events, but a specific role has not yet been identified.

Finally, several reports describe CLIC2 as a regulator of the ryanodine receptor, the major calcium-release channel of the sarcoplasmic reticulum [102-104]. CLIC2 is highly expressed in cardiac and skeletal muscle. CLIC2 binds directly to the ryanodine receptor and decreases its activity, and hence, $\mathrm{Ca}^{2+}$ release from the SR $[102,103]$. This interaction is modified by the redox state of CLIC2 and may represent a mechanism by which the redox state of the cell is linked to regulation of $\mathrm{Ca}^{2+}$ release and contractile activity of muscle [104]. It is important to note that although CLIC2 supports channel activity in vitro, the activity of CLIC2 in regulation of the ryanodine receptor is not thought to involve its channel function.

\section{CLICs as multifunctional proteins?}

A substantial body of evidence supports the hypothesis that CLICs can function as ion channels. Strong independent lines of evidence also support the hypothesis that CLICs possess other functions in the cell that appear to be unrelated to channel activity. One possibility is that CLICs are multi-functional proteins that can transition between membrane-inserted forms supporting ion channel activity, and soluble forms which may contribute to regulation of various cell processes such as structure of the cytoskeleton or modulation of gene expression. Thus, CLICs may act to integrate ion channel activities of intracellular membranes with regulation of cytoskeleton and/or corresponding gene expression. This model is reminiscent of the dual roles of $\beta$-catenin as a component of the cytoskeleton and as a regulator of transcription [105].

\section{Golgi pH Regulator}

The recently identified Golgi pH Regulator (GPHR) was discovered through a screen for mutants which affect Golgi function [106]. Mutation of this protein was shown to result in delayed transport of newly synthesized protein from the Golgi to the plasma membrane, impaired glycosylation of proteins along the exocytic pathway, and structural disorganization of the Golgi apparatus. GPHR was found to be targeted to the Golgi. Direct measurement of the steady state $\mathrm{pH}$ of intracellular compartments revealed that the mutation of GPHR was associated with decreased acidification of the cisternae of the Golgi and trans-Golgi network by 0.4 to $0.5 \mathrm{pH}$ units with no effect on lysosomal $\mathrm{pH}$ and no effect on endocytosis or recycling. Thus the absence of GPHR had a selective effect on Golgi acidification. Expression of recombinant GPHR followed by purification and reconstitution into planar lipid bilayers showed that GPHR functioned as a non-rectifying DIDS inhibitable, high conductance, weakly anion selective channel. These data strongly support the identification of GPHR as a conductive pathway that supports Golgi acidification. However, the experiments presented did not demonstrate altered chloride permeability of the Golgi or that the lack of acidification is due to absence of counterion conductance in the absence of GPHR. The possibility remains that GPHR may regulate Golgi pH by some other mechanism.

\section{Concluding Remarks}

The past two decades have witnessed an explosion of knowledge accompanied by ongoing controversy regarding chloride transport mechanisms of intracellular membranes.

Identification of newly recognized channel proteins, clarification of the relationships between chloride permeability and acidification, and the molecular description of previously mysterious 
human diseases all must be recognized as major accomplishments. Controversies include the longstanding problem of CFTR and intracellular acidification, and, perhaps most notably, the realization that intracellular $\mathrm{ClCs}$ are exchangers rather than channels. This stunning finding has forced a thorough reconsideration of some of the most fundamental assumptions about acidification of intracellular compartments, a reconsideration which is not yet fully resolved. Despite the advances, many fundamental questions persist: which proteins are responsible for which channel activities, how are these activities regulated, and exactly how do intracellular chloride channels contribute to integrated cell and organismal function. Are the intracellular $\mathrm{ClCs}$ responsible for counterion movement supporting acidification along the endosomal pathway or do they support acidification through some other mechanism? Does the absence of the actions of CFTR in intracellular membranes contribute to the cystic fibrosis disease phenotype, even if they do not regulate the $\mathrm{pH}$ of intracellular compartments? Are bestrophins channels, regulators of channels or both? Do CLICs really function as channels in intracellular membranes or is their true role something quite different? Undoubtedly more surprises lie head.

\section{Abbreviations}

$\begin{array}{ll}\text { CFTR } & \text { cystic fibrosis transmembrane conductance } \\ \text { GPHR } & \text { Golgi pH regulator } \\ \text { SR } & \text { sarcoplasmic reticulum } \\ \text { ER } & \text { endoplasmic reticulum }\end{array}$

\section{References}

[1]. Debska G, Kicinska A, Skalska J, Szewczyk A. Intracellular potassium and chloride channels: an update. Acta Biochim Pol 2001;48:137-44. [PubMed: 11440163]

[2]. Szewczyk A. The intracellular potassium and chloride channels: properties, pharmacology and function (review). Mol Membr Biol 1998;15:49-58. [PubMed: 9724922]

[3]. al-Awqati Q. Chloride channels of intracellular organelles. Curr Opin Cell Biol 1995;7:504-8. [PubMed: 7495569]

[4]. Carraro-Lacroix LR, Lessa LM, Fernandez R, Malnic G. Physiological implications of the regulation of vacuolar H+-ATPase by chloride ions. Braz J Med Biol Res 2009;42:155-63. [PubMed: 19274342]

[5]. Mellman I. The importance of being acid: the role of acidification in intracellular membrane traffic. J Exp Biol 1992;172:39-45. [PubMed: 1491231]

[6]. Laporte R, Hui A, Laher I. Pharmacological modulation of sarcoplasmic reticulum function in smooth muscle. Pharmacol Rev 2004;56:439-513. [PubMed: 15602008]

[7]. Thevenod F. Ion channels in secretory granules of the pancreas and their role in exocytosis and release of secretory proteins. Am J Physiol Cell Physiol 2002;283:C651-72. [PubMed: 12176723]

[8]. Haggie PM, Verkman AS. Defective organellar acidification as a cause of cystic fibrosis lung disease: reexamination of a recurring hypothesis. Am J Physiol Lung Cell Mol Physiol 2009;296:L859-67. [PubMed: 19329540]

[9]. Bauerfeind R, Huttner WB. Biogenesis of constitutive secretory vesicles, secretory granules and synaptic vesicles. Curr Opin Cell Biol 1993;5:628-35. [PubMed: 8257604]

[10]. Marshansky V, Ausiello DA, Brown D. Physiological importance of endosomal acidification: potential role in proximal tubulopathies. Curr Opin Nephrol Hypertens 2002;11:527-37. [PubMed: 12187318]

[11]. Wada Y, Sun-Wada GH, Tabata H, Kawamura N. Vacuolar-type proton ATPase as regulator of membrane dynamics in multicellular organisms. J Bioenerg Biomembr 2008;40:53-7. [PubMed: 18214654]

[12]. Forgac M. Vacuolar ATPases: rotary proton pumps in physiology and pathophysiology. Nat Rev Mol Cell Biol 2007;8:917-29. [PubMed: 17912264] 
[13]. al-Awqati Q, Barasch J, Landry D. Chloride channels of intracellular organelles and their potential role in cystic fibrosis. J Exp Biol 1992;172:245-66. [PubMed: 1337094]

[14]. Barasch J, Kiss B, Prince A, Saiman L, Gruenert D, al-Awqati Q. Defective acidification of intracellular organelles in cystic fibrosis. Nature 1991;352:70-3. [PubMed: 1712081]

[15]. Mulberg AE, Tulk BM, Forgac M. Modulation of coated vesicle chloride channel activity and acidification by reversible protein kinase A-dependent phosphorylation. J Biol Chem 1991;266:20590-3. [PubMed: 1657931]

[16]. Tamir H, et al. Secretogogue-induced gating of chloride channels in the secretory vesicles of parafollicular cells. Endocrinology 1994;135:2045-57. [PubMed: 7525261]

[17]. Barriere H, Bagdany M, Bossard F, Okiyoneda T, Wojewodka G, Gruenert D, Radzioch D, Lukacs GL. Revisiting the role of cystic fibrosis transmembrane conductance regulator and counterion permeability in the $\mathrm{pH}$ regulation of endocytic organelles. Mol Biol Cell 2009;20:3125-41. [PubMed: 19420138]

[18]. Chandy G, Grabe M, Moore HP, Machen TE. Proton leak and CFTR in regulation of Golgi pH in respiratory epithelial cells. Am J Physiol Cell Physiol 2001;281:C908-21. [PubMed: 11502568]

[19]. Lukacs GL, Chang XB, Kartner N, Rotstein OD, Riordan JR, Grinstein S. The cystic fibrosis transmembrane regulator is present and functional in endosomes. Role as a determinant of endosomal pH. J Biol Chem 1992;267:14568-72. [PubMed: 1378835]

[20]. Schapiro FB, Grinstein S. Determinants of the $\mathrm{pH}$ of the Golgi complex. J Biol Chem 2000;275:21025-32. [PubMed: 10748071]

[21]. Steinberg BE, Touret N, Vargas-Caballero M, Grinstein S. In situ measurement of the electrical potential across the phagosomal membrane using FRET and its contribution to the proton-motive force. Proc Natl Acad Sci U S A 2007;104:9523-8. [PubMed: 17517624]

[22]. Picollo A, Pusch M. Chloride/proton antiporter activity of mammalian CLC proteins ClC-4 and ClC-5. Nature 2005;436:420-3. [PubMed: 16034421]

[23]. Scheel O, Zdebik AA, Lourdel S, Jentsch TJ. Voltage-dependent electrogenic chloride/proton exchange by endosomal CLC proteins. Nature 2005;436:424-7. [PubMed: 16034422]

[24]. Gillespie D, Fill M. Intracellular calcium release channels mediate their own countercurrent: the ryanodine receptor case study. Biophys J 2008;95:3706-14. [PubMed: 18621826]

[25]. Pollock NS, Kargacin ME, Kargacin GJ. Chloride channel blockers inhibit Ca2+ uptake by the smooth muscle sarcoplasmic reticulum. Biophys J 1998;75:1759-66. [PubMed: 9746517]

[26]. Orci L, Ravazzola M, Storch MJ, Anderson RG, Vassalli JD, Perrelet A. Proteolytic maturation of insulin is a post-Golgi event which occurs in acidifying clathrin-coated secretory vesicles. Cell 1987;49:865-8. [PubMed: 3555846]

[27]. De Lisle RC, Williams JA. Zymogen granule acidity is not required for stimulated pancreatic protein secretion. Am J Physiol 1987;253:G711-9. [PubMed: 2447787]

[28]. O'Rourke B. Mitochondrial ion channels. Annu Rev Physiol 2007;69:19-49. [PubMed: 17059356]

[29]. Pedersen PL. Voltage dependent anion channels (VDACs): a brief introduction with a focus on the outer mitochondrial compartment's roles together with hexokinase-2 in the "Warburg effect" in cancer. J Bioenerg Biomembr 2008;40:123-6. [PubMed: 18780167]

[30]. Antonenko YN, Kinnally KW, Tedeschi H. Identification of anion and cation pathways in the inner mitochondrial membrane by patch clamping of mouse liver mitoplasts. J Membr Biol 1991;124:151-8. [PubMed: 1722254]

[31]. Sorgato MC, Keller BU, Stuhmer W. Patch-clamping of the inner mitochondrial membrane reveals a voltage-dependent ion channel. Nature 1987;330:498-500. [PubMed: 2446143]

[32]. Aon MA, Cortassa S, Maack C, O’Rourke B. Sequential opening of mitochondrial ion channels as a function of glutathione redox thiol status. J Biol Chem 2007;282:21889-900. [PubMed: 17540766]

[33]. De Marchi U, Szabo I, Cereghetti GM, Hoxha P, Craigen WJ, Zoratti M. A maxi-chloride channel in the inner membrane of mammalian mitochondria. Biochim Biophys Acta 2008;1777:1438-48. [PubMed: 18805391]

[34]. Mumbengegwi DR, Li Q, Li C, Bear CE, Engelhardt JF. Evidence for a superoxide permeability pathway in endosomal membranes. Mol Cell Biol 2008;28:3700-12. [PubMed: 18378695] 
[35]. Aon MA, Cortassa S, Marban E, O'Rourke B. Synchronized whole cell oscillations in mitochondrial metabolism triggered by a local release of reactive oxygen species in cardiac myocytes. J Biol Chem 2003;278:44735-44. [PubMed: 12930841]

[36]. De Angeli A, Monachello D, Ephritikhine G, Frachisse JM, Thomine S, Gambale F, Barbier-Brygoo $\mathrm{H}$. The nitrate/proton antiporter AtCLCa mediates nitrate accumulation in plant vacuoles. Nature 2006;442:939-42. [PubMed: 16878138]

[37]. Thompson RJ, Akana HC, Finnigan C, Howell KE, Caldwell JH. Anion channels transport ATP into the Golgi lumen. Am J Physiol Cell Physiol 2006;290:C499-514. [PubMed: 16403948]

[38]. Jentsch TJ. Chloride and the endosomal-lysosomal pathway: emerging roles of CLC chloride transporters. J Physiol 2007;578:633-40. [PubMed: 17110406]

[39]. Jentsch TJ. CLC chloride channels and transporters: from genes to protein structure, pathology and physiology. Crit Rev Biochem Mol Biol 2008;43:3-36. [PubMed: 18307107]

[40]. Plans V, Rickheit G, Jentsch TJ. Physiological roles of $\mathrm{CLC} \mathrm{Cl(-)/H} \mathrm{(+)} \mathrm{exchangers} \mathrm{in} \mathrm{renal}$ proximal tubules. Pflugers Arch 2009;458:23-37. [PubMed: 18853181]

[41]. Gunther W, Piwon N, Jentsch TJ. The ClC-5 chloride channel knock-out mouse - an animal model for Dent's disease. Pflugers Arch 2003;445:456-62. [PubMed: 12548389]

[42]. Wang SS, et al. Mice lacking renal chloride channel, CLC-5, are a model for Dent's disease, a nephrolithiasis disorder associated with defective receptor-mediated endocytosis. Hum Mol Genet 2000;9:2937-45. [PubMed: 11115837]

[43]. Gunther W, Luchow A, Cluzeaud F, Vandewalle A, Jentsch TJ. ClC-5, the chloride channel mutated in Dent's disease, colocalizes with the proton pump in endocytotically active kidney cells. Proc Natl Acad Sci U S A 1998;95:8075-80. [PubMed: 9653142]

[44]. Christensen EI, et al. Loss of chloride channel ClC-5 impairs endocytosis by defective trafficking of megalin and cubilin in kidney proximal tubules. Proc Natl Acad Sci U S A 2003;100:8472-7. [PubMed: 12815097]

[45]. Hara-Chikuma M, Wang Y, Guggino SE, Guggino WB, Verkman AS. Impaired acidification in early endosomes of ClC-5 deficient proximal tubule. Biochem Biophys Res Commun 2005;329:941-6. [PubMed: 15752747]

[46]. Hara-Chikuma M, Yang B, Sonawane ND, Sasaki S, Uchida S, Verkman AS. ClC-3 chloride channels facilitate endosomal acidification and chloride accumulation. J Biol Chem 2005;280:1241-7. [PubMed: 15504734]

[47]. Mohammad-Panah R, Wellhauser L, Steinberg BE, Wang Y, Huan LJ, Liu XD, Bear CE. An essential role for $\mathrm{ClC}-4$ in transferrin receptor function revealed in studies of fibroblasts derived from Clcn4-null mice. J Cell Sci 2009;122:1229-37. [PubMed: 19339555]

[48]. Kasper D, et al. Loss of the chloride channel ClC-7 leads to lysosomal storage disease and neurodegeneration. EMBO J 2005;24:1079-91. [PubMed: 15706348]

[49]. Graves AR, Curran PK, Smith CL, Mindell JA. The Cl-/H+ antiporter ClC-7 is the primary chloride permeation pathway in lysosomes. Nature 2008;453:788-92. [PubMed: 18449189]

[50]. Kornak U, et al. Loss of the ClC-7 chloride channel leads to osteopetrosis in mice and man. Cell 2001;104:205-15. [PubMed: 11207362]

[51]. Frattini A, et al. Chloride channel ClCN7 mutations are responsible for severe recessive, dominant, and intermediate osteopetrosis. J Bone Miner Res 2003;18:1740-7. [PubMed: 14584882]

[52]. Chu K, Snyder R, Econs MJ. Disease status in autosomal dominant osteopetrosis type 2 is determined by osteoclastic properties. J Bone Miner Res 2006;21:1089-97. [PubMed: 16813529]

[53]. Henriksen K, Gram J, Neutzsky-Wulff AV, Jensen VK, Dziegiel MH, Bollerslev J, Karsdal MA. Characterization of acid flux in osteoclasts from patients harboring a G215R mutation in ClC-7. Biochem Biophys Res Commun 2009;378:804-9. [PubMed: 19070589]

[54]. Hryciw DH, Ekberg J, Pollock CA, Poronnik P. ClC-5: a chloride channel with multiple roles in renal tubular albumin uptake. Int J Biochem Cell Biol 2006;38:1036-42. [PubMed: 16226913]

[55]. Hartzell HC, Qu Z, Yu K, Xiao Q, Chien LT. Molecular physiology of bestrophins: multifunctional membrane proteins linked to best disease and other retinopathies. Physiol Rev 2008;88:639-72. [PubMed: 18391176] 
[56]. Kunzelmann K, Kongsuphol P, Aldehni F, Tian Y, Ousingsawat J, Warth R, Schreiber R. Bestrophin and TMEM16-Ca(2+) activated $\mathrm{Cl}(-)$ channels with different functions. Cell Calcium 2009;46:233-41. [PubMed: 19783045]

[57]. Marmorstein LY, et al. The light peak of the electroretinogram is dependent on voltage-gated calcium channels and antagonized by bestrophin (best-1). J Gen Physiol 2006;127:577-89. [PubMed: 16636205]

[58]. Barro-Soria R, Aldehni F, Almaca J, Witzgall R, Schreiber R, Kunzelmann K. ER-localized bestrophin 1 activates $\mathrm{Ca}(2+)$-dependent ion channels TMEM16A and SK4 possibly by acting as a counterion channel. Pflugers Arch. 2009

[59]. Marmorstein AD, Kinnick TR. Focus on molecules: bestrophin (best-1). Exp Eye Res 2007;85:4234. [PubMed: 16720022]

[60]. Jouret F, et al. Cystic fibrosis is associated with a defect in apical receptor-mediated endocytosis in mouse and human kidney. J Am Soc Nephrol 2007;18:707-18. [PubMed: 17287432]

[61]. Ashley RH. Challenging accepted ion channel biology: p64 and the CLIC family of putative intracellular anion channel proteins (Review). Mol Membr Biol 2003;20:1-11. [PubMed: 12745921]

[62]. Cromer BA, Morton CJ, Board PG, Parker MW. From glutathione transferase to pore in a CLIC. Eur Biophys J 2002;31:356-64. [PubMed: 12202911]

[63]. Landry DW, Akabas MH, Redhead C, Edelman A, Cragoe EJ Jr. Al-Awqati Q. Purification and reconstitution of chloride channels from kidney and trachea. Science 1989;244:1469-72. [PubMed: 2472007]

[64]. Edwards JC, Kapadia S. Regulation of the bovine kidney microsomal chloride channel p64 by p59fyn, a Src family tyrosine kinase. J Biol Chem 2000;275:31826-32. [PubMed: 10930415]

[65]. Edwards JC, Tulk B, Schlesinger PH. Functional expression of p64, an intracellular chloride channel protein. J Membr Biol 1998;163:119-27. [PubMed: 9592076]

[66]. Duncan RR, Westwood PK, Boyd A, Ashley RH. Rat brain p64H1, expression of a new member of the p64 chloride channel protein family in endoplasmic reticulum. J Biol Chem 1997;272:23880 6. [PubMed: 9295337]

[67]. Qian Z, Okuhara D, Abe MK, Rosner MR. Molecular cloning and characterization of a mitogenactivated protein kinase-associated intracellular chloride channel. J Biol Chem 1999;274:1621-7. [PubMed: 9880541]

[68]. Valenzuela SM, et al. Molecular cloning and expression of a chloride ion channel of cell nuclei. J Biol Chem 1997;272:12575-82. [PubMed: 9139710]

[69]. Berryman M, Bruno J, Price J, Edwards JC. CLIC-5A functions as a chloride channel in vitro and associates with the cortical actin cytoskeleton in vitro and in vivo. J Biol Chem 2004;279:34794801. [PubMed: 15184393]

[70]. Cromer BA, et al. Structure of the Janus protein human CLIC2. J Mol Biol 2007;374:719-31. [PubMed: 17945253]

[71]. Littler DR, et al. The intracellular chloride ion channel protein CLIC1 undergoes a redox-controlled structural transition. J Biol Chem 2004;279:9298-305. [PubMed: 14613939]

[72]. Singh H, Ashley RH. CLIC4 (p64H1) and its putative transmembrane domain form poorly selective, redox-regulated ion channels. Mol Membr Biol 2007;24:41-52. [PubMed: 17453412]

[73]. Singh H, Cousin MA, Ashley RH. Functional reconstitution of mammalian 'chloride intracellular channels' CLIC1, CLIC4 and CLIC5 reveals differential regulation by cytoskeletal actin. FEBS J 2007;274:6306-16. [PubMed: 18028448]

[74]. Tulk BM, Kapadia S, Edwards JC. CLIC1 inserts from the aqueous phase into phospholipid membranes, where it functions as an anion channel. Am J Physiol Cell Physiol 2002;282:C110312. [PubMed: 11940526]

[75]. Tulk BM, Schlesinger PH, Kapadia SA, Edwards JC. CLIC-1 functions as a chloride channel when expressed and purified from bacteria. J Biol Chem 2000;275:26986-93. [PubMed: 10874038]

[76]. Warton K, et al. Recombinant CLIC1 (NCC27) assembles in lipid bilayers via a pH-dependent twostate process to form chloride ion channels with identical characteristics to those observed in Chinese hamster ovary cells expressing CLIC1. J Biol Chem 2002;277:26003-11. [PubMed: 11978800] 
[77]. Landry D, Sullivan S, Nicolaides M, Redhead C, Edelman A, Field M, al-Awqati Q, Edwards J. Molecular cloning and characterization of p64, a chloride channel protein from kidney microsomes. J Biol Chem 1993;268:14948-55. [PubMed: 7686908]

[78]. Heiss NS, Poustka A. Genomic structure of a novel chloride channel gene, CLIC2, in Xq28. Genomics 1997;45:224-8. [PubMed: 9339381]

[79]. Tulk BM, Edwards JC. NCC27, a homolog of intracellular Cl- channel p64, is expressed in brush border of renal proximal tubule. Am J Physiol 1998;274:F1140-9. [PubMed: 9841507]

[80]. Chuang JZ, Milner TA, Zhu M, Sung CH. A $29 \mathrm{kDa}$ intracellular chloride channel p64H1 is associated with large dense-core vesicles in rat hippocampal neurons. J Neurosci 1999;19:291928. [PubMed: 10191309]

[81]. Fernandez-Salas E, Sagar M, Cheng C, Yuspa SH, Weinberg WC. p53 and tumor necrosis factor alpha regulate the expression of a mitochondrial chloride channel protein. J Biol Chem 1999;274:36488-97. [PubMed: 10593946]

[82]. Berryman M, Bretscher A. Identification of a novel member of the chloride intracellular channel gene family (CLIC5) that associates with the actin cytoskeleton of placental microvilli. Mol Biol Cell 2000;11:1509-21. [PubMed: 10793131]

[83]. Edwards JC, Cohen C, Xu W, Schlesinger PH. c-Src control of chloride channel support for osteoclast $\mathrm{HCl}$ transport and bone resorption. J Biol Chem 2006;281:28011-22. [PubMed: 16831863]

[84]. Schlesinger PH, Blair HC, Teitelbaum SL, Edwards JC. Characterization of the osteoclast ruffled border chloride channel and its role in bone resorption. J Biol Chem 1997;272:18636-43. [PubMed: 9228032]

[85]. Ulmasov B, Bruno J, Woost PG, Edwards JC. Tissue and subcellular distribution of CLIC1. BMC Cell Biol 2007;8:8. [PubMed: 17326840]

[86]. Valenzuela SM, Mazzanti M, Tonini R, Qiu MR, Warton K, Musgrove EA, Campbell TJ, Breit SN. The nuclear chloride ion channel NCC27 is involved in regulation of the cell cycle. J Physiol 2000;529(Pt 3):541-52. [PubMed: 11195932]

[87]. Lubarsky B, Krasnow MA. Tube morphogenesis: making and shaping biological tubes. Cell 2003;112:19-28. [PubMed: 12526790]

[88]. Berry KL, Bulow HE, Hall DH, Hobert O. A C. elegans CLIC-like protein required for intracellular tube formation and maintenance. Science 2003;302:2134-7. [PubMed: 14684823]

[89]. Bohman S, Matsumoto T, Suh K, Dimberg A, Jakobsson L, Yuspa S, Claesson-Welsh L. Proteomic analysis of vascular endothelial growth factor-induced endothelial cell differentiation reveals a role for chloride intracellular channel 4 (CLIC4) in tubular morphogenesis. J Biol Chem 2005;280:42397-404. [PubMed: 16239224]

[90]. Tung JJ, Hobert O, Berryman M, Kitajewski J. Chloride intracellular channel 4 is involved in endothelial proliferation and morphogenesis in vitro. Angiogenesis 2009;12:209-20. [PubMed: 19247789]

[91]. Ulmasov B, Bruno J, Gordon N, Hartnett ME, Edwards JC. Chloride intracellular channel protein-4 functions in angiogenesis by supporting acidification of vacuoles along the intracellular tubulogenic pathway. Am J Pathol 2009;174:1084-96. [PubMed: 19197003]

[92]. Fernandez-Salas E, et al. mtCLIC/CLIC4, an organellular chloride channel protein, is increased by DNA damage and participates in the apoptotic response to p53. Mol Cell Biol 2002;22:3610-20. [PubMed: 11997498]

[93]. Ronnov-Jessen L, Villadsen R, Edwards JC, Petersen OW. Differential expression of a chloride intracellular channel gene, CLIC4, in transforming growth factor-beta1-mediated conversion of fibroblasts to myofibroblasts. Am J Pathol 2002;161:471-80. [PubMed: 12163372]

[94]. Shukla A, Malik M, Cataisson C, Ho Y, Friesen T, Suh KS, Yuspa SH. TGF-beta signalling is regulated by Schnurri-2-dependent nuclear translocation of CLIC4 and consequent stabilization of phospho-Smad2 and 3. Nat Cell Biol 2009;11:777-84. [PubMed: 19448624]

[95]. Suh KS, et al. Antisense suppression of the chloride intracellular channel family induces apoptosis, enhances tumor necrosis factor $\{$ alpha $\}$-induced apoptosis, and inhibits tumor growth. Cancer Res 2005;65:562-71. [PubMed: 15695400] 
[96]. Suh KS, et al. CLIC4 mediates and is required for Ca2+-induced keratinocyte differentiation. J Cell Sci 2007;120:2631-40. [PubMed: 17636002]

[97]. Shi Y, Massague J. Mechanisms of TGF-beta signaling from cell membrane to the nucleus. Cell 2003;113:685-700. [PubMed: 12809600]

[98]. Myers K, Somanath PR, Berryman M, Vijayaraghavan S. Identification of chloride intracellular channel proteins in spermatozoa. FEBS Lett 2004;566:136-40. [PubMed: 15147883]

[99]. Ponsioen B, van Zeijl L, Langeslag M, Berryman M, Littler D, Jalink K, Moolenaar WH. Spatiotemporal regulation of chloride intracellular channel protein CLIC4 by RhoA. Mol Biol Cell 2009;20:4664-72. [PubMed: 19776349]

[100]. Berryman MA, Goldenring JR. CLIC4 is enriched at cell-cell junctions and colocalizes with AKAP350 at the centrosome and midbody of cultured mammalian cells. Cell Motil Cytoskeleton 2003;56:159-72. [PubMed: 14569596]

[101]. Shanks RA, Larocca MC, Berryman M, Edwards JC, Urushidani T, Navarre J, Goldenring JR. AKAP350 at the Golgi apparatus. II. Association of AKA P350 with a novel chloride intracellular channel (CLIC) family member. J Biol Chem 2002;277:40973-80. [PubMed: 12163479]

[102]. Board PG, Coggan M, Watson S, Gage PW, Dulhunty AF. CLIC-2 modulates cardiac ryanodine receptor Ca2+ release channels. Int J Biochem Cell Biol 2004;36:1599-612. [PubMed: 15147738]

[103]. Dulhunty AF, Pouliquin P, Coggan M, Gage PW, Board PG. A recently identified member of the glutathione transferase structural family modifies cardiac RyR2 substate activity, coupled gating and activation by Ca2+ and ATP. Biochem J 2005;390:333-43. [PubMed: 15916532]

[104]. Jalilian C, Gallant EM, Board PG, Dulhunty AF. Redox potential and the response of cardiac ryanodine receptors to CLIC-2, a member of the glutathione S-transferase structural family. Antioxid Redox Signal 2008;10:1675-86. [PubMed: 18522493]

[105]. Brembeck FH, Rosario M, Birchmeier W. Balancing cell adhesion and Wnt signaling, the key role of beta-catenin. Curr Opin Genet Dev 2006;16:51-9. [PubMed: 16377174]

[106]. Maeda Y, Ide T, Koike M, Uchiyama Y, Kinoshita T. GPHR is a novel anion channel critical for acidification and functions of the Golgi apparatus. Nat Cell Biol 2008;10:1135-45. [PubMed: 18794847] 